\title{
Aplicación del Test de Sprint en la preparación física de los aspirantes a árbitros profesionales de fútbol
}

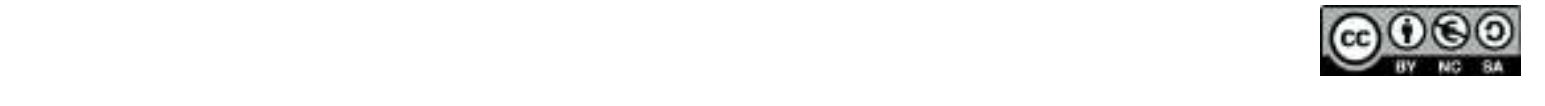

Application of the Sprint Test in the physical preparation of aspiring professional football referees

Diego Xavier Borja Urquizo ${ }^{1}$, Olmedo Javier Mármol Escobar ${ }^{2}$, Beto Orlando Arcos Ortiz ${ }^{3}$ \& Marco Antonio Camacho Escobar ${ }^{4}$

Recibido: 10-02-2019 / Revisado: 15-02-2019 /Aceptado: 04-03-2019/ Publicado: 14-06-2019

\begin{abstract}
.
DOI: https://doi.org/10.33262/cienciadigital.v3i2.5.541

The present work has as objective, to apply the Test of Sprint for the physical preparation of the aspirants to professional referees of soccer of the province of Bolivar. By means of the investigation, it was possible to determine the process of the physical preparation in two moment's pre and post test. It was worked with a mixed approach of applied type, with a sample of 28 participants, by means of the application of photoelectric cells to measure the maximum speed that the aspiring referees can make, the application of the test of sprint was made in progressive form, by means of repetitions of races of going and return of $40 \mathrm{~m}$, with periods of rest of 10 seconds, after each rest increased progressively the rhythm. Thanks to the results obtained, it is possible to mention that in the first take the physical preparation obtained results of a

\footnotetext{
${ }^{1}$ Maestrante. Entrenamiento Deportivo. Universidad Estatal de Bolívar. Guaranda. Ecuador. Docente Unidad Educativa San Miguel de los Bancos. Distrito 17D12 Puerto Quito. diegox.borja@educacion.gob.ec.

${ }^{2}$ Docente de Cuarto Nivel. Director del Departamento de Cultura Física. Universidad Estatal de Bolívar. Guaranda. Ecuador. omarmol@ueb.edu.ec

${ }^{3}$ Unidad Educativa Joaquín Lalama 18H00090. Distrito 18D02C02-07. Docente de Cultura Física. Ambato. Ecuador.18h00090@gmail.com

${ }^{4}$ Decano de la Facultad Ciencias de la Educación. Universidad Estatal de Bolívar. Guaranda, Ecuador. marancames59@yahoo.com
} 
regular and insufficient performance in the aspiring referees whose values represent $92.86 \%$; while in the second take, six months later, the results improved significantly and a rating scale of $78.00 \%$ between good and very good was obtained. It is concluded that the capacity of acceleration in the process of physical preparation must maintain a continuous training to improve the physical preparation of the aspiring football referees in the province of Bolivar.

Keywords: Physical preparation, performance, test sprint, referee.

\section{Resumen.}

El presente trabajo tiene como objetivo, aplicar el Test de Sprint para la preparación física de los aspirantes a árbitros profesionales de fútbol de la provincia de Bolívar. Mediante la investigación se pudo determinar el proceso de la preparación física en dos momentos pre y post test. Se trabajó con un enfoque mixto de tipo aplicado, con una muestra de 28 participantes, mediante la aplicación de células fotoeléctricas para medir la velocidad máxima que pueden realizar los aspirantes a árbitros, la aplicación del test de sprint fue realizada en forma progresiva, mediante repeticiones de carreras de ida y vuelta de $40 \mathrm{~m}$, con periodos de descanso de 10 segundos, luego de cada descanso aumentó progresivamente el ritmo. Gracias a los resultados obtenidos, se puede mencionar que en la primera toma la preparación física obtuvo resultados de un rendimiento regular e insuficiente en los aspirantes a árbitros cuyos valores representan el 92,86 \%; mientras que en la segunda toma, seis meses después, los resultados mejoraron de forma significativa y se obtuvo una escala valorativa de 78,00 \% entre bueno y muy bueno. Se concluye que la capacidad de aceleración en el proceso de preparación física debe mantener un entrenamiento continuo para mejorar la preparación física de los aspirantes a árbitros de fútbol en la provincia de Bolívar.

Palabras claves: Preparación física, rendimiento, test sprint, árbitro.

\section{Introducción.}

Según la FIFA 2014 citado por Weston (2015), en el fútbol siempre debe haber un árbitro, el mismo que también puede ser denominado como "referí", y es la persona encargada de que el reglamento se cumpla en un partido, debe dar constancia de todo lo sucedido en el encuentro, además tiene la potestad de hacer cumplir el reglamento antes de que inicie el encuentro e incluso posterior al partido, siempre y cuando las acciones se estén desarrollando dentro del terreno de juego. Si el árbitro principal considera necesario, puede disponer de dos árbitros adicionales o asistentes, éstos deben situarse fuera del terreno de juego, sin embargo, éstos también tienen el deber de controlar y amonestar las acciones que no estén correctas, 
en función al reglamento. Hay casos en los que se necesita un cuarto árbitro, pero éste sólo sustituye en caso de que alguno de los tres designados previamente sufra alguna lesión o surja algún inconveniente. $\mathrm{Y}$ finalmente casos excepcionales como en los mundiales, cuenta con un quinto árbitro que apoya al equipo de árbitros encargados de un encuentro deportivo.

Los árbitros de campo, conjuntamente con los árbitros asistentes son los responsables directos de mantener controlados a los jugadores y sus comportamientos, a la vez aplicar durante el juego las reglas correspondientes al mismo (Castillo, Cámara, \& Yanci, 2016). Es decir, el orden y el cumplimiento de las reglas dependen en gran manera de los árbitros encargados del encuentro.

Según Reina y Hernández (2012) el árbitro es la persona encargada en mantener el orden durante un encuentro deportivo, además de cumplir y hacer cumplir las reglas del juego que se practican, es decir el árbitro es una persona preparada en el tema de deportes, por lo general los árbitros deben especializarse en un solo deporte, en este caso de estudio, se centra en los árbitros de fútbol, quienes son los responsables de que un evento deportivo sea desarrollado con orden(total normalidad).

El proceso de formación arbitral está constituido por diferentes aspectos, tales como: el conocimiento de las leyes que rigen el arbitraje deportivo tanto nacional como internacionalmente; el entrenamiento físico, el cual permite desarrollar condiciones físicas, técnicas y tácticas para mejorar el rendimiento (Ninanya y Sierra, 2017). Todas estas características son fundamentales para formar un buen árbitro, el cual contará con las condiciones necesarias para tener una participación justa e imparcial, teniendo la capacidad de enjuiciar correctamente una acción, interpretar lo que sucede y comparar con las exigencias del reglamento es decir evaluar el conocimiento interpretado según las reglas existentes en forma imparcial.

De acuerdo con Patiño \& Cañadas (2015), los sistemas de preparación física que se utilizan actualmente no facilitan viabilidad de ejercicios en la rutina diaria de entrenamiento, el cual se convierte en un entrenamiento sin procesos y con poca fundamentación científica, generando deficiencias en la coordinación, equilibrio, movilidad, velocidad, resistencia, flexibilidad y fuerza. La mayoría de los entrenadores físicos no disponen de sistemas de planificación fundamentados en una metodología científica, sino más bien efectúan una preparación física general, es decir, solo en base de experiencias vividas, lo que da como resultado el bajo rendimiento en la conducción de los partidos. Esta problemática se evidencia notoriamente en la escasa participación de árbitros ecuatorianos en torneos internacionales, ya que no logran superar las evaluaciones físicas, teóricas y médicas.

\section{Revisión de la literatura.}

Casa (2016) trabajó en la investigación "La preparación física en el rendimiento del árbitro del fútbol profesional en el campeonato nacional de fútbol del Ecuador", cuyo propósito fue 
determinar la importancia de la preparación física, para dicho estudio aplicó un test a los 219 árbitros de fútbol del Ecuador. Por otra parte, Merino (2014) realizó una investigación sobre "La preparación física en el rendimiento arbitral de los integrantes de la asociación de árbitros profesionales de fútbol de Pastaza", analizando la preparación física y el desempeño de los árbitros integrantes de la asociación en Pastaza; quienes concluyen sobre la falta de capacitación de los preparadores físicos de 5 asociaciones provinciales, por lo que sugieren un manual de métodos teóricos y prácticos para la preparación física de los árbitros del fútbol ecuatorianos.

La preparación física es un pilar fundamental conjuntamente con la preparación psicológica y teórica, la sinergia de estos elementos ayudan a que el árbitro cumpla un buen rol cuando es parte de un encuentro deportivo (Quintero, 2017), a su vez Pazmiño (2017), menciona que la preparación física conjuga el aprovechamiento de un conjunto de técnicas o medios que aumentan las posibilidades de triunfar y ayuda a incrementar los niveles de rendimiento físico, es decir, el desarrollo que el individuo logra a nivel individual, lo puede conjugar con un equipo y conseguir metas grupales, siempre y cuando todo el equipo también se preocupe por su preparación física.

El motivo de la preparación física en los aspirantes a árbitros profesionales es formar árbitros con alta resistencia, pues una de las principales capacidades que debe desarrollar específicamente es la resistencia, para que pueda recorrer el terreno de juego mientras dure el encuentro deportivo y supervisar todos y cada uno de los movimientos realizados por los participantes, por lo cual es necesario e indispensable que un árbitro posea un estado físico óptimo, pues si esto se cumple, el árbitro puede seguir de cerca las acciones del juego y con ello evitar al máximo los errores de apreciación (Ninanya \& Sierra, 2017).

El rendimiento arbitral exige ejercicios aeróbicos y anaeróbicos, el trote como ejercicio aeróbico para oxigenar el sistema cardiovascular y genera un beneficio a corto plazo, debido que promueve un mejor rendimiento; mientras el ejercicio anaeróbico más utilizado es la velocidad porque permite el desplazamiento del árbitro para tomar la decisión correcta en cuanto a la dirección del juego. La preparación psicológica es fundamental, pues la euforia es una característica predominante en un encuentro deportivo, el manejo a nivel psicológico es necesario para poder mantener la calma frente a las adversidades que se presentan en dichos encuentros, el equilibrio psicológico ayuda en gran manera para tolerar, tomar decisiones equitativas y firmes. Un plan de entrenamiento físico debe complementarse de manera obligatoria con un entrenamiento psicológico, pues de esa manera se logra un desarrollo integral (Paredes, 2017).

De acuerdo con Acosta, Sanabria, \& Agudelo (2016), la resistencia es la capacidad para realizar un esfuerzo eficaz y mantenerlo durante el mayor tiempo posible. Los principales órganos del cuerpo que intervienen en un buen proceso de resistencia son: los pulmones y el corazón, de los primeros emana el oxígeno para todo el cuerpo, el segundo bombea la sangre 
a cada rincón del cuerpo y permite que la persona se mantenga en movimiento. La principal característica física de un árbitro es la resistencia, pues debe desplazarse continuamente por el área donde se desarrolla el juego, por ello es necesario que cuente con una preparación física adecuada y una resistencia física óptima (Campos, 2017). Esta característica está estrechamente ligada a otras capacidades físicas como la velocidad y la fuerza, para un mejor desempeño.

Como eje transversal se toma en cuenta la preparación física, pues los inadecuados sistemas de entrenamiento producen deficiencia en el desarrollo de las actitudes físicas, cansancio muscular y lesiones y un deficiente desempeño profesional (Barros, 2018).

De acuerdo a la revisión de la literatura, el cansancio físico influye en el desempeño arbitral, el cual está ligado con la disminución del rendimiento físico, por esta razón es fundamental una actualización de conocimientos sobre las reglas del juego, mientras que en el ámbito deportivo es indispensable la preparación física y mental del árbitro, por ello el objetivo que plantea el presente estudio fue determinar la importancia de la preparación física en el rendimiento arbitral de los aspirantes a árbitros profesionales de fútbol de la provincia de Bolívar.

\section{Metodología.}

El presente estudio presenta un enfoque mixto cuantitativo-cualitativo: cuantitativo pues se realizó una recolección de datos numéricos mediante la estadística descriptiva, pues fue necesario recolectar datos numéricos o cuantificables de forma secuencial y probatoria, estos sirven para realizar el análisis estadístico y comprobar las hipótesis, con el fin de demostrar teorías y establecer modelos de conducta. Este tipo de estudios, se utiliza para consolidar una teoría o un estudio previamente realizado (Hernández-Sampieri, Fernández, \& Baptista, 2014). Cualitativo porque se recolectó información en forma de cualidades, información simbólica verbal y escrita, mediante un plan de recolección de datos, realizando preguntas de investigación antes, durante o después de la recolección de datos (Hernández-Sampieri et al., 2014).

El presente estudio tuvo una investigación aplicada, pues se realizaron dos test a los participantes, para verificar su condición física antes y después.

\section{Alcance}

El alcance del estudio fue definido en función del tiempo programado, con el cual se pudo identificar la condición física de los aspirantes a árbitros profesionales en la provincia de Bolívar, el trabajo de campo fue determinado para seis meses, concordando con Mocha Bonilla J., (2018) quien menciona que se debe realizar una toma al inicio, posteriormente luego de seis meses la segunda toma, de tal manera que se pueda realizar un análisis y 
comparación entre los dos resultados para verificar si hubo o no mejoría en los sujetos de estudio. En la tabla 1. Se puede observar a los participantes del estudio.

Tabla 1. Población de estudio

\begin{tabular}{ll} 
Provincia & Bolívar \\
\hline Institución & Asociación de árbitros profesionales de provincia de \\
Participantes & Bolívar \\
& 28
\end{tabular}

Fuente: Elaboración propia.

Dentro de esta investigación se tomó como muestra a los aspirantes a árbitros profesionales de la provincia Bolívar quienes fueron evaluados en las canchas del colegio "Unidad Educativa Guaranda" donde realizan sus entrenamientos.

\section{Instrumentos y protocolos}

El instrumento a utilizar para la recolección de datos fue el Test Físico que se realizó a los aspirantes, específicamente en este caso se aplicó el Test Sprint.

Se aplicó de la siguiente manera:

- Los tiempos de las carreras se registraron con puertas de cronometraje electrónicos (células fotoeléctricas) las puertas de cronometraje se colocaron a una distancia no mayor a $100 \mathrm{~m}$ del suelo, también se realizó la prueba con un cronometro manual.

- La puerta de salida se colocó en el punto 0m y la puerta de llegada en el punto de $40 \mathrm{~m}$, la línea de salida se marcó a $1.5 \mathrm{~m}$ antes de la puerta de salida.

- Los árbitros se alinearon para salir tocando la línea de salida con el pie delantero, una vez dada la orden de salida, el árbitro empieza el Test.

- Los árbitros tuvieron 60 segundos como máximo para recuperarse entre cada una de las carreras de 40m, los árbitros regresaron caminando para su recuperación.

\section{Resultados.}

En primera instancia es necesario conocer los resultados obtenidos antes de la aplicación del test Sprint. A continuación, se presentan:

Tabla 2. Resultados pre-test Sprint

Condición
Muy Buena
Buena
Regular
Insuficiente
Total

Frecuencia

$0 \quad 0,00 \%$

$2 \quad 7,14 \%$

$23-82,14 \%$

$3 \quad 10,72 \%$

$28 \quad 100 \%$

Fuente: Elaboración propia. 
Gráfico 1. Resultados pre-test Sprint

\section{PRIMERA MUESTRA}

ГBuena $\square$ Regular $\square$ Insuficiente

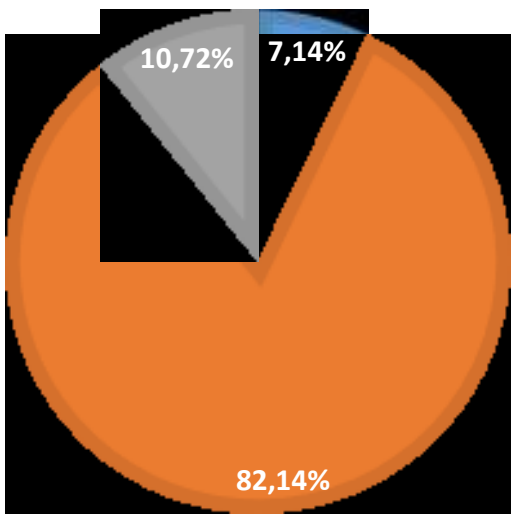

Fuente: Elaboración propia.

El 7,14\% de los aspirantes a árbitros presentan una escala valorativa de bueno, el 82,14\% tienen una escala valorativa de regular, y un $10,72 \%$ una escala valorativa de insuficiente; Sumado los valores más bajos se tiene un porcentaje de $92,86 \%$.

De acuerdo al análisis de los datos se obtuvo una escala valorativa, la cual da un total de $92,86 \%$ en el nivel más bajo del rendimiento físico en los aspirantes a árbitros profesionales, indicando un rendimiento inadecuado.

A continuación se indican los resultados obtenidos de la aplicación del post-test Sprint:

Tabla 3. Resultados pre-test Sprint

\begin{tabular}{lll} 
Condición & Frecuencia & Porcentaje \\
\hline Muy Buena & 6 & $21,50 \%$ \\
Buena & 16 & $57,00 \%$ \\
Regular & 6 & $21,50 \%$ \\
Insuficiente & 0 & $0,00 \%$ \\
Total & 28 & $100 \%$ \\
& Fuente: Elaboración propia.
\end{tabular}


Gráfico 2. Resultados post-test Sprint

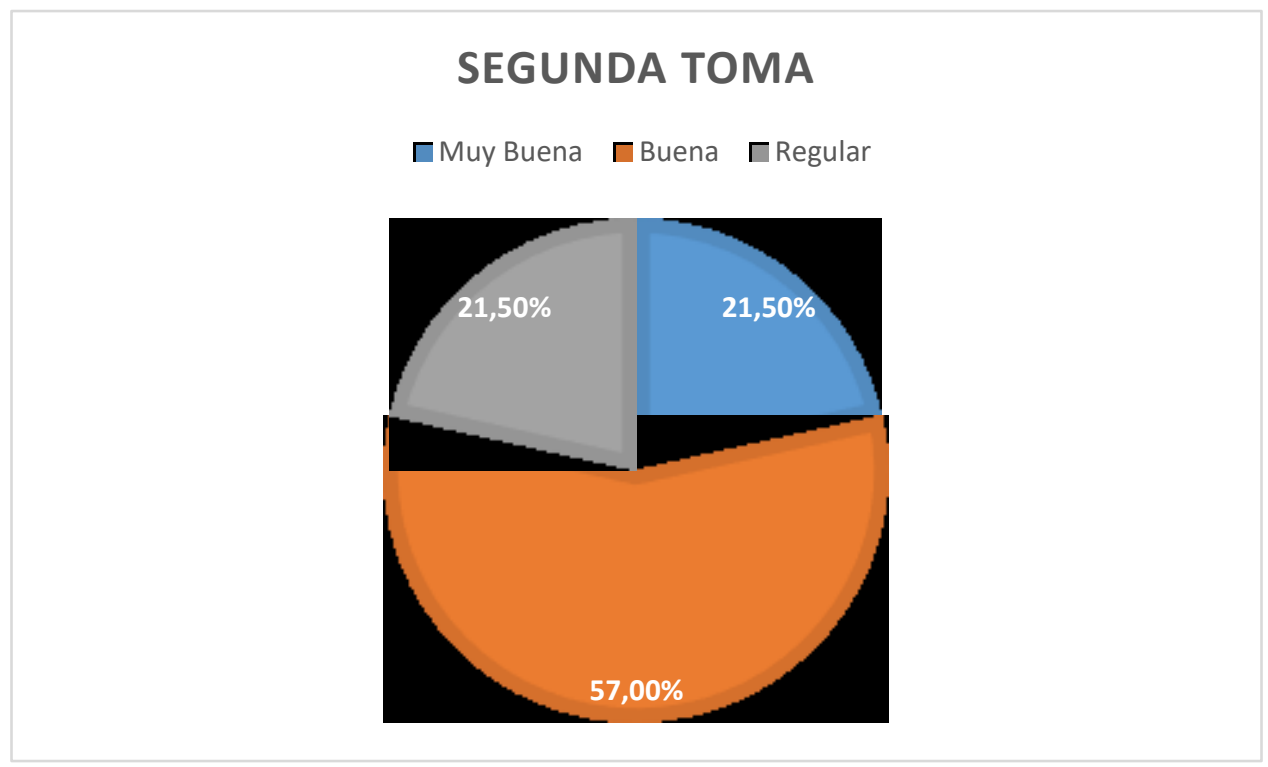

Fuente: Elaboración propia.

El 21,5\% de los aspirantes a árbitros participantes tienen una escala valorativa de regular, el $57 \%$ tienen una escala valorativa de buena, y un $21,5 \%$ una escala valorativa de muy buena; Sumado los valores más altos se tiene un porcentaje de $78,5 \%$.

De acuerdo al análisis de los datos se obtuvo una escala valorativa, la cual da un total de 78,5 $\%$ en el nivel más alto del rendimiento físico de los aspirantes a árbitros profesionales, indicando un rendimiento significativo después de aplicar el test de sprint posterior a los seis meses.

\section{Conclusiones.}

Al tratarse de un estudio preliminar, se puede mencionar que las principales características que distinguen a un buen árbitro son: el conocimiento teórico del deporte que va a supervisar, la habilidad de tomar decisiones, un rendimiento físico óptimo, la capacidad de resistencia, una comunicación asertiva-efectiva y un equilibrio psicológico. La preparación física en el rendimiento arbitral de los aspirantes a árbitros profesionales de fútbol es muy importante, pues en base los resultados obtenidos en los test se evidenciaron que es necesario mejorar el desarrollo de sus capacidades físicas, cuyos resultados expuestos abren futuras líneas de investigación para emprender nuevos estudios. 
El nivel de preparación física de los aspirantes a árbitros profesionales de fútbol de la provincia de Bolívar fue regular e insuficiente, necesitando una aplicación metodológica mediante ejercicios aeróbicos y anaeróbicos, los mismos que ayudan en la mejora de la condición física.

Después de la aplicación del Test de Sprint el nivel de rendimiento subió considerablemente en relación al pre-test, observando niveles de bueno y muy bueno, confirmando que una correcta y preparada planificación física da como resultado un aumento del nivel de rendimiento en el grupo de estudio, pues la capacidad de aceleración en el proceso de preparación física debe mantener un entrenamiento continuo para mejorar el rendimiento físico de los aspirantes a árbitros de fútbol en la provincia de Bolívar.

\section{Referencias bibliográficas.}

Acosta, P., Sanabria, Y., \& Agudelo, C. (2016). Desarrollo de la resistencia en jugadores de fútbol: método intermitente vs método continuo. Revista Actividad Física Y Desarrollo Humano, 7(2), 1-9.

Barros, F. (2018). La preparación fisica y las lesiones deportivas en los integrantes de la asociación de árbitros profesionales de fútbol de Pastaza. Universidad Técnica de Ambato. https://doi.org/10.22201/fq.18708404e.2004.3.66178

Campos, J. (2017). Factores determinantes del arbitraje en fútbol: Análisis de los árbitros de la Región de Murcia Murcia. Universidad Católica de Murcia.

Casa, S. (2016). La preparación fisica en el rendimiento del árbitro del fútbol profesional en el campeonato nacional de fútbol del Ecuador. Universidad Técnica de Ambato. https://doi.org/10.15517/ap.v29i119.18693

Castillo, D., Cámara, J., \& Yanci, J. (2016). Análisis de las respuestas físicas y fisiológicas de árbitros y árbitros asistentes de fútbol durante partidos oficiales de Tercera División de España. RICYDE: Revista Internacional de Ciencias Del Deporte, 11(45), 250-261. https://doi.org/10.5232/ricyde

Hernández-Sampieri, R., Fernández, C., \& Baptista, M. (2014). Metodología de la investigación (6ta ed.). México: McGraw-Hill.

Merino, J. (2014). La preparación física en el rendimiento arbitral de los integrantes de la asociación de árbitros profesionales de fútbol de Pastaza. Universidad Técnica De Ambato. https://doi.org/10.1007/s10584-009-9544 
Mocha Bonilla, J. A. (2018). Análisis del consumo máximo de oxígeno (vo2máx) post intervención de un programa en juegos pre-deportivos. Ciencia Digital, 2(2), 229244. doi:https://doi.org/10.33262/cienciadigital.v2i2.85

Ninanya, C., \& Sierra, M. (2017). Perfil antropométrico y aptitud física de los áritros de fútbol pertenecientes a la provincia de Huancayo. Universidad Nacional del Centro del Perú.

Paredes, C. (2017). La preparación física y su incidencia en el rendimiento arbitral de los integrantes del gremio CADASP del cantón Pelileo provincia de Tungurahua. Las tareas scolares y su incidencia en el rendimiento académico. Universidad Técnica de Ambato. https://doi.org/10.15517/ap.v29i119.18693

Patiño, Ó., \& Cañadas, M. (2015). Análisis de la figura del árbitro deportivo y su intervención en el proceso de formación deportiva. Revista Pedagógica de Educación Física ADAL, $18(30), 25-32$.

Pazmiño, R. (2017). El rendimiento físico en el desempeño arbitral de la asociación nacional de árbitros de baloncesto del Ecuador. Universidad Técnica de Ambato.

Quintero, F. (2017). Programa de entrenamiento para mejorar la condición física resistencia general en los árbitros de fútbol playa. Universidad de Guayaquil.

Reina, A., \& Hernández, A. (2012). Revisión de indicadores de rendimiento en fútbol. Revista Iberoamericana de Ciencias de La Actividad Física Y El Deporte, 1(1), 1-14. https://doi.org/10.24310/riccafd.2012.v1i1.1990

Weston, M. (2015). Match performances of soccer referees: the role of sports science. Movement \& Sport Sciences - Science \& Motricité, 87(7), 113-117. https://doi.org/10.1051/sm/2014011

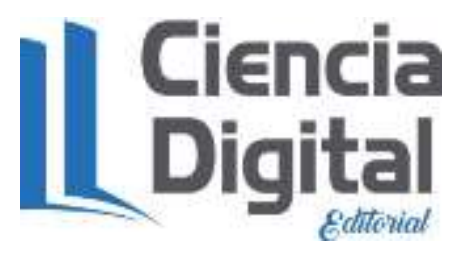




\section{PARA CITAR EL ARTÍCULO INDEXADO.}

Borja Urquizo, D., Mármol Escobar, O., Arcos Ortiz, B., \& Camacho Escobar, M. (2019). Aplicación del Test de Sprint en la preparación física de los aspirantes a árbitros profesionales de fútbol. Ciencia Digital, 3(2.5), 165-175. https://doi.org/10.33262/cienciadigital.v3i2.5.541

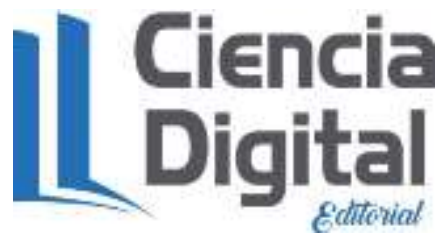

El artículo que se publica es de exclusiva responsabilidad de los autores y no necesariamente reflejan el pensamiento de la Revista Ciencia Digital.

El artículo queda en propiedad de la revista y, por tanto, su publicación parcial y/o total en otro medio tiene que ser autorizado por el director de la Revista Ciencia Digital.
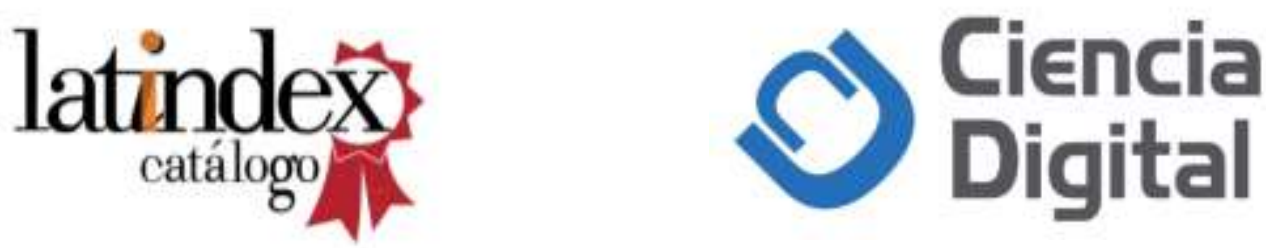